\title{
"EACH OF US, IN OUR OWN WAY, WAS BROKEN": GÉNERO, VULNERABILIDAD Y COMUNIDAD EN MAD MAX: FURY ROAD
}

\author{
IRINA CRUZ PEREIRA \\ ADHUC-Centre de Recerca Teoria, Gènere, Sexualitat \\ Universitat de Barcelona \\ irina.cp91@gmail.com
}

\section{RESUMEN}

En el mundo distópico representado en Mad Max: Fury Road (2015) la violencia se manifiesta a través de la explotación de la vulnerabilidad de los cuerpos de los personajes. A partir de la descentralización del héroe de acción masculino y la introducción de una heroína protagonista, en el filme se propone la desestabilización de las normas de género convencionales del cine de acción. Furiosa, la heroína, se construye a sí misma como sujeto inmune para sobrevivir en la sociedad hipermasculina de Citadel, pero deviene vulnerable al establecer lazos con el resto de los personajes. De la misma forma, el protagonista masculino, Max, pasa por el reconocimiento progresivo de su propia vulnerabilidad y la aceptación de la necesidad de exponerse como cuerpo a otros como vía de supervivencia. Como se mostrará en este artículo, la experiencia compartida de la vulnerabilidad y del duelo abren en Mad Max: Fury Road un lugar común en el que se rechazan los valores hipermasculinos y donde se valora una idea de comunidad fundamentada en la vulnerabilidad como intrínseca a la condición humana.

PALABRAS CLAVE: Género, Violencia, Vulnerabilidad, Comunidad, Hipermasculinidad, Cine de Acción.

\section{“EACH OF US, IN OUR OWN WAY, WAS BROKEN": GENDER, VULNERABILITY AND COMMUNITY IN MAD MAX: FURY ROAD}

\section{ABSTRACT}

In the dystopian world represented in Mad Max: Fury Road violence manifests itself through the exploitation of the vulnerability of the character's bodies. Through the decentralization of the male action hero and the introduction of a female protagonist, the film proposes the destabilization of conventional gender norms in action cinema. Furiosa, the heroine, constructs herself as an immune subject in order to survive within the hypermasculine society of Citadel, but she also becomes vulnerable by establishing bonds with the rest of the characters. In the same way, the male protagonist, Max, goes through the progressive recognition of his own vulnerability and his bodily exposure to others as a means of survival. As I will show in this article, the characters' shared experience of vulnerability and grief in Mad Max: Fury Road opens up a common place in which hypermasculine values are rejected and an idea of community based on vulnerability as intrinsic to the human condition is valued.

KEYWORDS: Gender, Violence, Vulnerability, Community, Hypermasculinity, Action Cinema.

\section{INTRODUCCIÓN}

Mad Max: Fury Road (2015) es la cuarta película de la saga distópica que cuenta la historia de Max Rockatansky, creada por el director australiano George Miller. 
En 1979, Miller estrenó la primera entrega, Mad Max, con Mel Gibson interpretando a su torturado protagonista. La premisa de la saga ha sido explicada como: "civilization has failed, and it has failed because of deeply rooted problems in the organization and character of society" (Sharrett 1985: 82).

El Max de Mel Gibson es un policía que lucha por mantener el orden en los primeros años de decaída de la civilización, tras el colapso de la industria del petróleo. El primer filme se inscribe en la ficción de venganza, ${ }^{1}$ con Max perdiendo a su compañero, su mujer y su hijo a manos de una brigada de delincuentes. Tres años después, Miller estrena Mad Max 2: The Road Warrior (1981), en la que el protagonista se enfrenta al caos civil que obliga a los ciudadanos a migrar a The Wasteland, la extensa área completamente árida del centro de Australia. En la misma línea, en Mad Max 3: Beyond Thunderdome (1985), el protagonista ayuda a huir a una tribu de niños que sueña con viajar al mundo pre-apocalíptico que consideran un mito. Pese a conseguir salvarlos, llevándolos a las ruinas de Sídney, Max sigue vagando exiliado en el desierto.

Las tres películas de la saga de George Miller se inscriben en el contexto del cine popular en la década de 1980, analizado por Susan Jeffords (1994). ${ }^{2}$ De acuerdo con Jeffords, se trata de una época de transición de la figura del justiciero, típica de los años 1970, al hard body de la década de los 1980. La autora define al justiciero como un héroe que "battles alone against an increasingly deteriorating society in which the only recourse from crime, violence and corruption is the determined individual who acts on his own principles and commitments" (1994: 17). ${ }^{3}$ En contraste con la figura del justiciero, Jeffords sitúa los hard bodies, es decir "heroes men who are pitted against bureaucracies that have lost touch with the people they are to serve" (1994: 19). Max, como héroe, evoluciona desde el policía que emprende una violenta batalla personal contra el crimen hasta convertirse en el líder de los rebeldes que luchan por defenderse de las autoridades corruptas, y en este sentido ejemplifica la transición descrita por Jeffords.

En este artículo se analiza la cuarta película de la saga, Mad Max: Fury Road (Miller 2015), en la que el director muestra cómo, cincuenta años después del Apocalipsis, la civilización australiana ha desaparecido por completo. Immortan Joe (Hugh Keays-Byrne), el tirano que controla la distribución del bien más preciado en el desierto, el agua, tiene preso a Max (Tom Hardy). El protagonismo del filme se desvía así a Furiosa (personaje interpretado por Charlize Theron), la

1 En la traducción al español de "avenger narrative" o "western narrative", el término "narrativa" no resulta igual de consolidado que en el original inglés, por lo que en este estudio se opta por "ficción", un término que cuenta con connotaciones transmediales y sigue la lógica en la denominación de géneros como la ciencia ficción o la road fiction.

${ }^{2}$ Pese a ser películas de producción australiana, la saga de Mad Max sigue las convenciones del cine de acción hollywoodense descritas por Jeffords.

${ }^{3}$ Jeffords utiliza, entre otros, el personaje interpretado por Clint Eastwood en Dirty Harry (Siegel, 1971) para ejemplificar la figura del justiciero. 
única mujer que sirve al ejército del tirano. Max se ve involucrado en la lucha de Furiosa por liberar a las esclavas sexuales de Immortan Joe, que concluye con el asesinato del tirano y la esperanza de reconstrucción de la ciudad bajo valores no patriarcales. Una vez más, tras el desenlace Max se retira del grupo para seguir merodeando por el desierto.

Así, a partir de la descentralización del héroe de acción masculino y la introducción de una heroína protagonista, en Mad Max: Fury Road se propone la desestabilización de las normas de género convencionales del cine de acción. Recurriendo al pensamiento de Judith Butler sobre la vulnerabilidad, la pérdida y el duelo, así como la producción del género mediante la distribución desigual de la vulnerabilidad (Torras Francés y Gama Leyva 2014: 100-101), este artículo mostrará cómo Furiosa, la heroína, se construye a sí misma como sujeto inmune para sobrevivir en la sociedad hipermasculina de Citadel, pero deviene vulnerable al establecer lazos con el resto de los personajes. De la misma forma, el protagonista masculino, Max, pasa por el reconocimiento progresivo de su propia vulnerabilidad y la aceptación de la necesidad de exponerse como cuerpo a otros como vía de supervivencia. Estas experienciacias compartidas abren en Mad Max: Fury Road un lugar común en el que se rechazan los valores hipermasculinos y donde se valora una idea de comunidad fundamentada en la vulnerabilidad como intrínseca a la condición humana.

\section{(HIPER)MASCULINIDAD EN LA SAGA MAD MAX}

Teniendo en cuenta la estrecha relación entre la vulnerabilidad y el género, es útil considerar cómo se ha manifestado y analizado la masculinidad del héroe protagonista en las anteriores entregas de la saga, y qué aspectos se han conservado o alterado en el arco evolutivo del personaje en el cuarto filme. A partir de la concepción de las masculinidades como "forms in which many dynamics of violence take shape" de R. W. Connell (2000: 224), y su reflexión sobre la masculinidad hegemónica (ligada a la violencia, la confrontación y la dominación) y formas alternativas, "more open to negotiation, cooperation and equality" (2000: 224), Galina Hanley interpreta Mad Max (1979) como un ejemplo de performance de masculinidades violentas, y afirma que el miedo de Max a convertirse en un monstruo, abandonando la relación con su familia, es el motor de la trama del filme (Hanley 2010). Katherine Biber, por su parte, ve esta evolución del personaje hacia la violencia como un símbolo de la inviabilidad del ideal de la masculinidad hegemónica: “Living and dying by the rules of the road, these men have no outlet, no escape. [...] Trapped on the murderous highways, men like Max (the journeyman actor; everyman) confirm the inescapability of failure, the impossibility of masculinity" (2001: 38). Los dos argumentos cobran sentido si se piensa en la historia de Max en esta primera película como la inversión de la tradicional ficción de venganza, en la que las acciones violentas del héroe tienen como objetivo recuperar la masculinidad vulnerada $\mathrm{y}$, como 
señala Cornell, la fantasía de control (2009: 121). En Mad Max (1979) esta venganza no llega hasta el final de la película y, en lugar de reafirmar la masculinidad heróica, supone su caída en el círculo de violencia y el horror. Max se muestra durante el filme como vulnerable y, al mismo tiempo, turbado por su propia agresividad:

MAX. I'm scared, Fif. You know why? It's that rat circus out there. I'm beginning to enjoy it.

FIFI. What is this, bonny week?

MAX. Look, any longer out on that road and I'm one of them, you know? A terminal crazy... only I got a bronze badge to say I'm one of the good guys. (Miller 1979)

Max decide irse de vacaciones con su familia, pero no consigue vencer al círculo violento que poco a poco lo va absorbiendo. Mad Max relata cómo el protagonista se transforma en el estereotipo de masculinidad agresiva, brutal y tóxica que película tras película convierte en inhabitable The Wasteland, la zona árida de Australia en la que se sitúa la ficción.

En Mad Max 2 (1981) Miller presenta un entorno mucho más severo y bárbaro que en su primer filme, con personajes deshumanizados y movidos por su agresividad y egoísmo. Frente a ello, la película relata el proceso de rehumanización de Max a través de su vínculo con la tribu de niños que encuentra en el desierto, abriendo la veda a la oposición entre hipermasculinidad y comunidad vulnerable que será explorada en Mad Max Beyond Thunderdome (Miller 1985) y, sobre todo, en Mad Max: Fury Road (Miller 2015).

La hipermasculinidad ha sido definida por Donald L. Mosher y Silvan S. Tomkins (1988) como la exageración del estereotipo de género masculino. El índice que crearon para medirla determina tres componentes: la actitud encallecida en relación con el sexo, la visión del peligro como algo excitante y la percepción de la violencia como manifestación primitiva de masculinidad. Jennifer Anne Hall (en Scharrer 2001: 617) añade que los hombres con esta actitud tienden a inhibir los afectos relacionados con la sensibilidad y codificados convencionalmente como femeninos, particularmente el miedo o la compasión. Burk, Burkhart y Sikorski redefinen la concepción de la hipermasculinidad en base a los trabajos anteriores, buscando una mayor consideración del papel de las relaciones de poder y reconociendo la vinculación entre el miedo, la rabia y la violencia interpersonal (Burk, Burkhart y Sikorski 2004: 5). Los autores ofrecen así una definición más amplia:

Hypermasculinity is a construct that describes men who exhibit an exaggeration of the traditional male gender role, including characteristics such as a supervaluation of competitive, aggressive activities and devaluation of cooperative, care-taking activities. In general, status and pride are highly valued, self-reliance or mistrust of others is evident, violence is perceived as necessary to maleness, women are predominantly seen as sexual objects or conquests, and sensation seeking is vital to life. Additionally, the expressed emotions of these individuals are likely constricted to displays of anger, disgust, and 
contempt, whereas the expression of emotions such as fear or love is perceived as weak. (2004: 14)

A partir de esta teorización se analiza aquí la representación de la (hiper)masculinidad en Mad Max: Fury Road, y la relación que se muestra entre ésta, la violencia y la vulnerabilidad de sus personajes.

\section{VULNERABILIDAD EXPLOTADA}

MAX. My name is Max. My world is fire and blood. Once, I was a cop. A road warrior searching for a righteous cause. As the world fell, each of us, in our own way, was broken. It was hard to know who was more crazy, me or everyone else. (Mad Max: Fury Road, 2015)

El mundo de Mad Max: Fury Road está construido sobre los pilares de la violencia y la agresividad. La performance hipermasculina ${ }^{4}$ de los personajes en la película lleva al extremo las prácticas asociadas a la violencia: se lucha tanto por el agua y la gasolina como por la sangre y la leche humana, todos fluidos esenciales para sobrevivir en un mundo destruido por la avaricia y la codicia de poder. El personaje de Nux (Nicholas Hoult), uno de los miembros del ejército de Immortan Joe, es ejemplar para demostrar la relación entre el culto a Immortan Joe, la violencia y la performance de la hipermasculinidad que define al tirano y a sus guerreros. En una escena marcada por el entusiasmo y el fervor, Nux pelea agresivamente con su compañero y fantasea con morir en la batalla para ganarse un puesto en Walhalla, el supuesto paraíso que espera a los guerreros tras la muerte: "If I'm gonna die, I'm gonna die historic on the Fury Road" (Miller 2015). Cuando a Nux le dicen que no puede luchar por estar enfermo, él exclama: "I'm not staying here dying soft!" (Miller 2015), lo que evoca la oposición entre el hard body del guerrero y el soft body vulnerable (Jeffords 1994). Una de las principales características de la hipermasculinidad es la independencia o desconfianza en otros, de nuevo esencial en el comportamiento de los guerreros, si bien con un matiz: Nux es desde el principio de la película dependiente de la sangre de Max. Pese a que Max no es visto en este punto del filme como un ser humano sino como una simple bolsa de sangre (hasta el final de la película los personajes se refieren a él como "Bolsa de Sangre"), el arco evolutivo del personaje de Nux se basa en el reconocimiento de esta dependencia, de su humanidad. Rechazado por el líder, es capaz de establecer vínculos con el resto de los personajes, a partir de la confianza mutua. Sin embargo, esta vulnerabilidad que consigue reconocer Nux es explotada de forma violenta por el entorno. Esta violencia es percibida en

\footnotetext{
${ }^{4}$ La performance hipermasculina se comprende aquí a partir de Judith Butler, quien teoriza en su obra sobre la cualidad performativa de los roles de género, desnaturalizándolos y señalando su carácter constructivo (2002; 2007), y define el género como una "fantasía instaurada y circunscrita en la superficie de los cuerpos" (2007: 267). Véase también Tasker (1995) sobre la representación de la masculinidad en el cine de acción.
} 
la hipermasculinidad como necesaria (Burk et al. 2004: 14), y en el mundo de Mad Max no sólo está presente, sino que compone la base sobre la que se construye la sociedad.

En Fury Road, más que en las anteriores películas de la saga, se entiende la violencia de la forma en que la define Fina Birulés cuando afirma que:

La violencia humana es fruto de la voluntad de controlar el cuerpo o los desplazamientos de la víctima, de la voluntad de que nada altere o interfiera en la supuesta soberanía, invulnerabilidad y completitud del sujeto, como si éste reaccionara agresivamente cuando, en sus relaciones y conflictos con los demás, entrevé su propia vulnerabilidad y dependencia o cuando en su entorno alguien se singulariza o irrumpe una dinámica de forma inesperada. (2007: 19; énfasis en el original)

Las palabras de la filósofa se adecuan con exactitud a la dinámica de poder que se establece en la película entre Immortan Joe y los personajes que lo desafían. En la primera escena del filme, Max es capturado por el ejército de Immortan Joe sin más objetivo que el de la explotación reificadora de su cuerpo: es reducido, atado, tatuado y quemado. Le arrebatan los símbolos con los que se identifica a Max en los anteriores filmes: su coche y la chaqueta de cuero. La escena de su captura puede ser interpretada como metáfora de una violación, tanto a nivel visual como narrativo, ya que alude al imaginario de la violación ejercida contra los personajes femeninos en el cine (Max silenciado con la mordaza, forzado e inmovilizado sobre la mesa, penetrado por la aguja del tatuador). Miller utiliza a Max para representar no sólo la violencia que caracteriza la atmósfera de Citadel sino también la historia de Furiosa, cuando nos enseña la marca de Immortan Joe en la nuca de ésta tras ver a Max sufrir el mismo destino. El espectador no precisa así de flashbacks para conocer el pasado de Furiosa, ya que Miller lo cuenta a través del cuerpo humillado y vulnerable del héroe.

La película relata, de forma literal, los esfuerzos de Joe por controlar el cuerpo y los desplazamientos de Max, Furiosa y las mujeres, fundamentando su trama en una persecución de ida y vuelta entre agresor y víctimas. Siguiendo a Birulés en la cita anterior, Furiosa se singulariza de la masa e interrumpe la dinámica de control de Immortan Joe para liberar los cuerpos de las mujeres y el suyo propio, sacándolos físicamente del círculo de dominio del tirano. Como señala Judith Butler:

El cuerpo supone mortalidad, vulnerabilidad, praxis: la piel y la carne nos exponen a la mirada de los otros, pero también al contacto y la violencia, y también son cuerpos los que nos ponen en peligro de convertirnos en agentes e instrumento de todo esto [...]. El cuerpo tiene una dimensión invariablemente pública. (Butler 2006: 52)

Es esta dimensión pública de los cuerpos lo que les convierte en vulnerables. Resulta muy sugerente la idea de la exposición de la piel y la carne propuesta por Butler si la pensamos en relación con la representación de la vulnerabilidad en la película. En la escena de presentación de Immortan Joe se muestra cómo un 
personaje vulnerable se autoconstruye una fachada de inmunidad hipermasculina: niños soplan polvo blanco sobre su piel, enferma y herida, colocan una armadura rígida sobre su pecho y hombros, protegiéndole y mostrando las insignias de batalla que lo revalidan como líder, y, finalmente, colocan el símbolo del imperio sobre su pelvis (haciendo referencia al carácter patriarcal de su autoridad). Él mismo coloca sobre su cara una máscara que ofrece un aspecto aterrador, pero de la cual es dependiente para respirar. El cuerpo de Joe es extremadamente frágil, pero se torna a sí mismo rígido e inviolable cada vez que se debe exponer públicamente [fig. 1]. En el extremo opuesto están sus esposas, cuyos cuerpos han existido siempre como inermes. Al principio de la película las mujeres apenas cubren sus cuerpos (aunque cubrirlos, en su situación, no las convertiría en inmunes), pero conforme avanza la película los propios personajes se atavían con nuevos trapos, ropajes y accesorios, símbolo de su adaptación al nuevo entorno y la progresiva resistencia a la explotación de su vulnerabilidad, alejándose de una performance de la hiperfeminidad como sumisa.

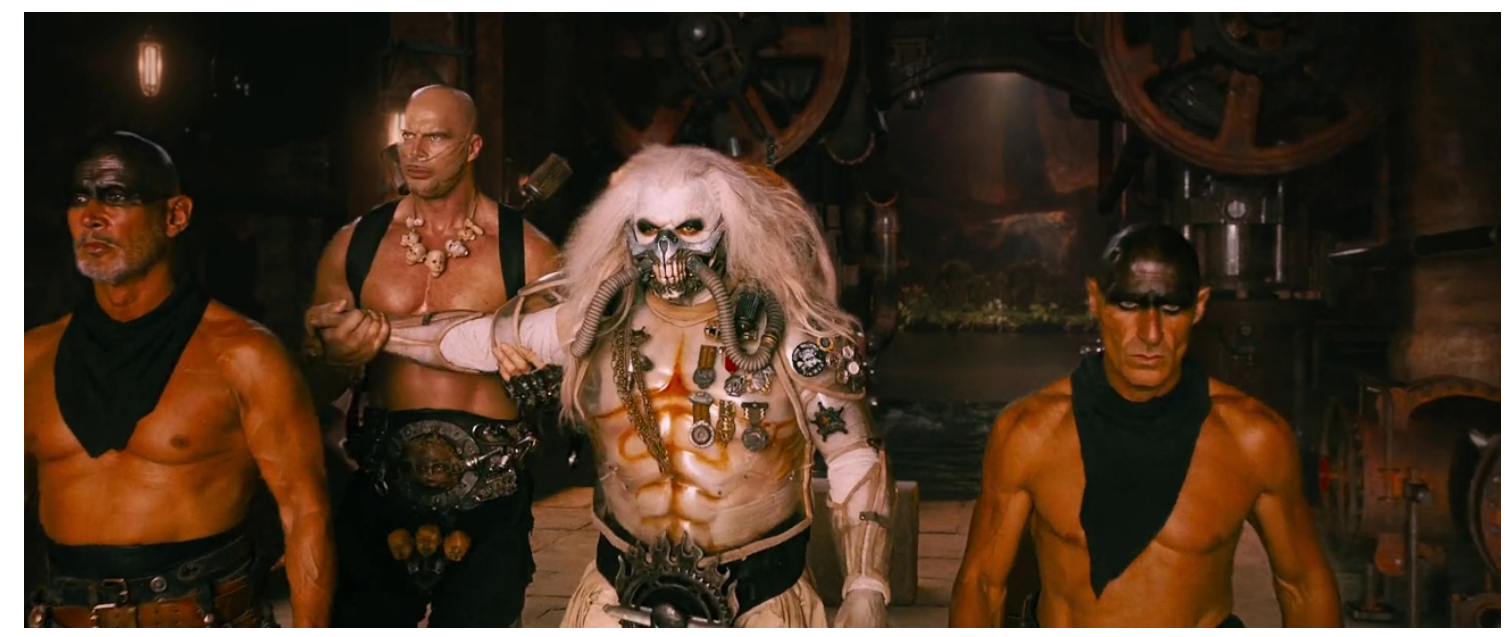

Fig. 1: Fotograma de Mad Max: Fury Road (2015)

Butler afirma que "la violencia consiste siempre en la explotación de ese lazo original, de esa forma original por la que existimos, como cuerpo, fuera de nosotros y para otros" (2006: 54). Es así como se representa la violencia en Fury Road, a partir de la explotación de la vulnerabilidad de los personajes a través de sus cuerpos. Max tan sólo es considerado útil por su sangre, al ser donante universal, y se utiliza al frente del coche de Nux como emblema, símbolo externo del espíritu de lucha, ya que él mismo proporciona la sangre que Nux necesita para mantenerse fuerte en batalla [fig. 2]. Max no es capturado para cumplir la función del guerrero, predeterminada a los hombres bajo el mandato de Immortan Joe, sino que su cuerpo es instrumentalizado para producir vida en lugar de muerte (Freitas 2015). Los cuerpos de las mujeres, de la misma forma, se reducen a su función reproductora, ya sea produciendo una descendencia sana al líder o leche maternal para alimentar a los nuevos cuerpos de la ciudadela. De 
esta manera, la película pone de manifiesto la dimensión pública del cuerpo, así como el vínculo entre la distribución de la vulnerabilidad y el género, como se analizará en la siguiente sección.

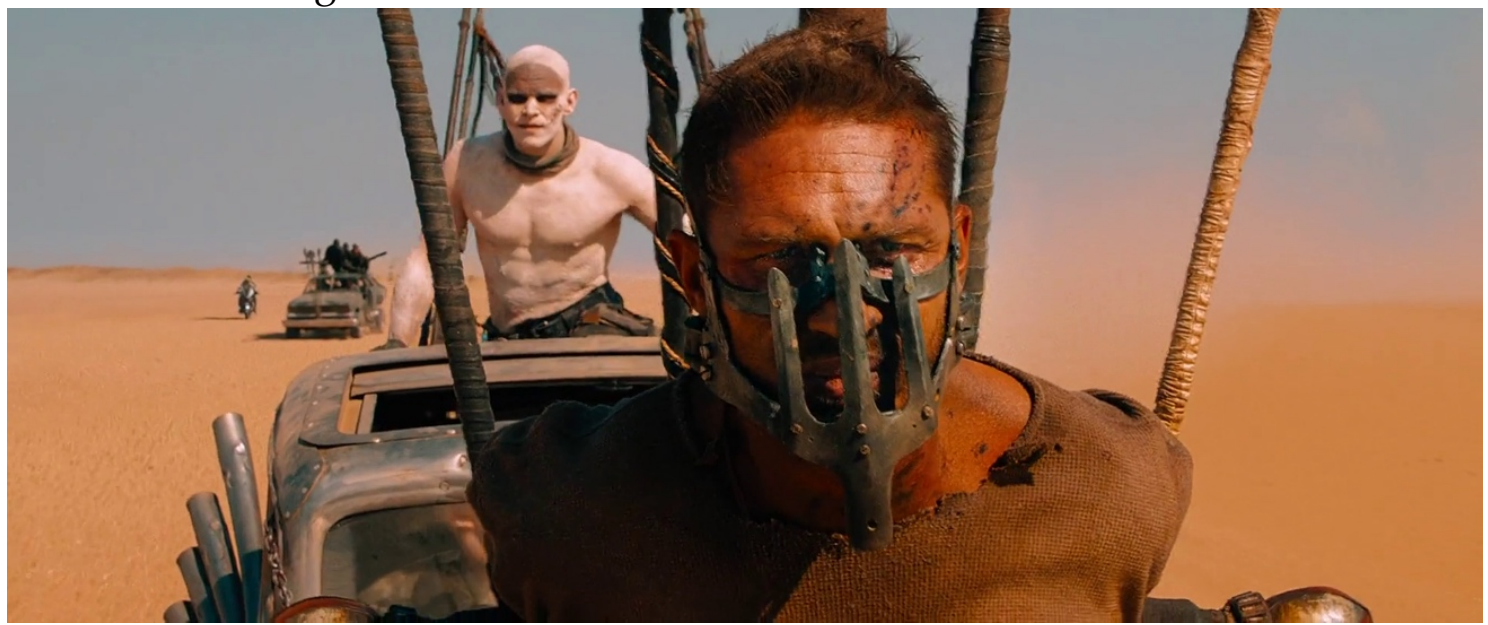

Fig. 2: Fotograma de Mad Max: Fury Road (2015)

\section{CONSTRUCCIÓN DEL GÉNERO EN BASE A LA VULNERABILIDAD}

A partir del diálogo entre Judith Butler y Adriana Cavarero, recogido en Cuerpo, memoria y representación (Saez Tajafuerce 2014), Meri Torras y Michelle Gama reflexionan en torno a la forma en que se construyen la masculinidad y la feminidad, es decir:

[...] a través de la distribución de la vulnerabilidad. Esta distribución desigual es parte del proceso de la producción y la regulación del género. Si se reduce la vulnerabilidad a un atributo femenino o la invulnerabilidad a un atributo masculino [...] se institucionaliza (y naturaliza) el problema en lugar de abrirlo a una comprensión crítica. (Torras y Gama 2014: 100-101)

A diferencia de las convenciones del cine de acción, que suelen codificar la masculinidad como inmune y la feminidad como inerme, Fury Road combina en la representación de la vulnerabilidad de los personajes de Max y Furiosa rasgos tradicionalmente asociados a ambos géneros. El arco de evolución de los dos protagonistas pasa por reconocer la vulnerabilidad que les ha sido explotada violentamente y, así, formar una comunidad basada en la exposición y el vínculo con el otro.

Andrea Braithwaite afirma que las imágenes de la masculinidad que exponen su fragilidad permiten problematizar las propias construcciones culturales de género (Braithwaite 2011). Es este el caso de Max, que se representa desde el inicio de la película como vulnerable, en contraposición a la aparente dureza impenetrable de Immortan Joe. La escena de apertura del filme le presenta como un personaje solitario, deteriorado física y mentalmente. Con el cuerpo y la cara cubiertos con harapos consigue una apariencia dura, pero huye al sentirse amenazado. Max es rápidamente despojado de sus ropas y afeitado 
por el ejército de Immortan Joe, y la exposición de su cuerpo desnudo e inmovilizado evidencia su vulnerabilidad. Tom Hardy, el actor que lo interpreta, remarca el valor que aporta a su interpretación la aceptación de la vulnerabilidad por parte de Max:

That's a symbiotic theme throughout George's Wasteland. It costs a human being. There's pain. And it's technically very uncool to be a superhero that hurts. But if you smash your face into the dirt, you have to play it. This is tough, so I make it look tough. That really earthed it for me, to physically accept vulnerability. (CR 2015)

La presentación de los personajes de Max e Immortan Joe funciona como introducción de los motivos de Furiosa para rebelarse contra el régimen tiránico. Pese a tratarse de un personaje fuerte, física y psicológicamente, el pasado de Furiosa la marca como vulnerable, habiendo sido desde niña víctima del régimen de Immortan Joe. Durante la primera mitad de la película Furiosa pinta su rostro con grasa negra ocultándolo, lo que resulta significativo considerando la importancia que Miller le dedica a los ojos y la mirada de Furiosa como vía de representación de su faceta vulnerable. Eric Whipp, el colorista de la película, explica cómo el director insistió en que los ojos de Theron fueran alterados en postproducción para exagerar su contraste y color con este objetivo (Heusser 2015) [fig. 3].

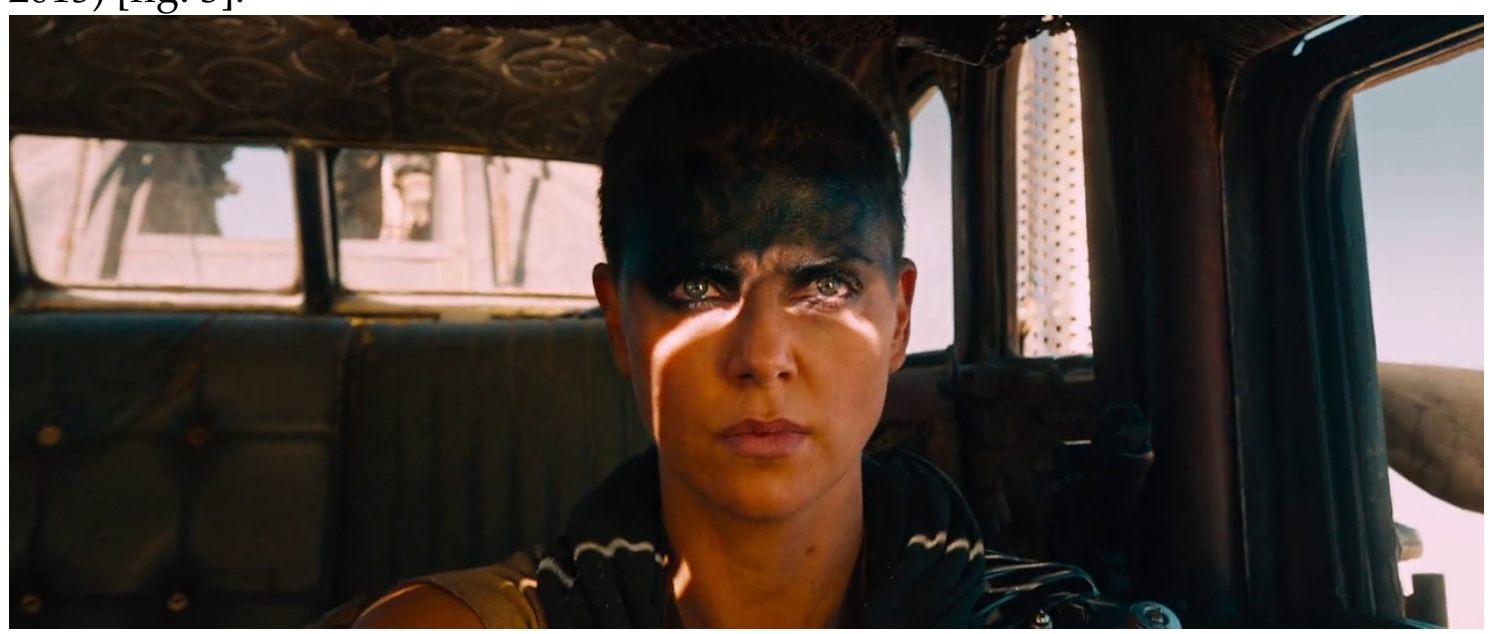

Fig. 3: Fotograma de Mad Max: Fury Road (2015)

El brazo prostético de Furiosa también funciona como símbolo de su esfuerzo por permanecer inmune y es en los momentos en los que no cuenta con él cuando se muestra más vulnerable, siendo el brazo una muestra de su (hipermasculina) fuerza. Es el caso de su primer encuentro con Max, cuando se ve amenazada y lucha violentamente por defender su plan de huida. En la escena en que se enfrenta a su imposible regreso utópico al descubrir que ya no existe The Green Place, el lugar donde nació y creía encontrar refugio, es ella misma la que se deshace del brazo protésico. Este es el momento de la película en el que se muestra más inerme física y emocionalmente. Finalmente, Furiosa pierde su prótesis en el momento en que mata a Immortan Joe, deshaciéndose de la 
necesidad de aparentar rigidez e inmunidad hipermasculinas, así como de la idea del cuerpo como total y autónomo.

Planteando al héroe como vulnerable de principio a fin, Fury Road no obliga a Max a reconstruirse como hard body tras el quebrantamiento de su fachada al principio de la película, sino que le confiere un arco evolutivo basado en el progresivo reconocimiento de la vulnerabilidad y de la necesidad de exponerse como cuerpo a los otros para sobrevivir. Furiosa sigue el mismo camino, construyéndose a sí misma como sujeto inmune en el marco de la sociedad hipermasculina de Citadel, pero deshaciéndose de dicha construcción conforme avanza la trama del filme.

\section{“WE KEEP MOVING": PÉRDIDA Y DUELO EN COMÚN}

Los protagonistas de Fury Road reciben nombres relacionados con la idea de furor o ira exaltada, que puede manifestarse en su aspecto más cercano a la locura, como es el caso del "mad" que acompaña a Max durante toda la saga, o la "furia" en el caso de Furiosa (y del propio título de la película). En esta cuarta entrega Max se encuentra rebajado a la supervivencia y aislado del resto de la humanidad. En el caso de Furiosa, se trata de un personaje movido por la rabia. Como afirma Hannah Arendt, la rabia brota como emoción política cuando "es ofendido nuestro sentido de la justicia" (2006: 85). De acuerdo con Butler, tanto la rabia como el dolor por la pérdida llevan al sujeto a una posición "afuera de uno mismo", lo que la autora denomina una posición de sujeto "ex-tático":

\footnotetext{
"Ex-tático" significa, literalmente, estar afuera de uno mismo, y puede tener varios sentidos: ser transportado por una pasión más allá de uno mismo, pero también estar fuera de sí de rabia o de dolor. Pienso que si todavía puedo dirigirme a un "nosotros" o incluirme en sus términos, es porque estoy hablándoles a aquellos de nosotros que están viviendo en cierto modo fuera de sí, se trate de pasión sexual, de pena o de furia política. (2006: 50)
}

Así, tanto Max como Furiosa evolucionan durante la ficción convirtiéndose en sujetos ex-táticos, movidos por la rabia y el dolor de la pérdida, y capaces de vivir fuera de sí, expuestos, y de establecer lazos relacionales a partir del reconocimiento de su vulnerabilidad.

El duelo es de igual manera un tema transversal en el filme. Max, como personaje, se construye a partir de sus pérdidas: no sólo la de su trabajo, su mujer y su hijo, sino la de todo un mundo que ha visto reducirse a la barbarie. Fury Road abre con Max perseguido por la memoria de aquellos a quienes ha perdido. "I tell myself they cannot touch me. They are long dead. I am the one who runs from the living and the dead" (Miller 2015), narra el protagonista en el monólogo inicial. Furiosa parte de un lugar muy similar, reaccionando contra los culpables de que hubiese perdido tanto a su madre como al resto de la comunidad que sentía como propia, la formada por las Vuvalini of Many Mothers. Antes de ser secuestrada por Immortan Joe, Furiosa vivía con ellas en The Green Place, una de 
las pocas zonas fértiles en The Wasteland. Las Vuvalini mantenían allí una sociedad formada aparentemente de manera exclusiva por mujeres, en la que criaban a sus hijas de forma comunitaria. Furiosa es arrebatada de su entorno siendo una niña y llevada a Citadel, donde Immortan Joe impone unos valores completamente opuestos a los de su comunidad original.

Así, el arco de ambos personajes pasa por el duelo como reconocimiento de la propia vulnerabilidad, de un estado ex-tático que, en palabras de Fina Birulés, "consigue formar un tenue nosotros" (2007: 21). Butler reflexiona sobre el potencial relacional del duelo, afirmando que:

Cuando perdemos a ciertas personas o cuando hemos sido despojados de un lugar o de una comunidad [...] algo acerca de lo que somos se nos revela, algo que dibuja los lazos que nos ligan a otro, que nos enseña que estos lazos constituyen lo que somos, los lazos o nudos que nos componen. (2006: 48)

A partir de la experiencia individual y compartida del duelo se establecen los vínculos entre los protagonistas de Fury Road. Max vive aislado, acostumbrado a sobrevivir por sus propios medios y a no confiar en nadie. Entra en la historia de Furiosa como antagonista, obstaculizando su camino de forma violenta, aterrorizado y desesperado por salvarse una vez más. Pero pronto su actitud se vuelve receptiva: acepta la ayuda de Furiosa para quitarse la mordaza y la confianza que ella deposita en él al enseñarle el mecanismo secreto del camión de guerra.

El duelo de Max se representa a través de las alucinaciones que sufre, en las que las personas que ha perdido desde que comenzó su viaje le persiguen. En el primer acto de la película están muy presentes, cuando Max se encuentra solo ante las amenazas, e incluso le impiden avanzar y salvarse. A partir de su acercamiento a Furiosa y a su grupo desaparecen las alucinaciones, pues ha encontrado a alguien con quien compartir el dolor de la pérdida. Cuando decide separarse de ellas y seguir su propio camino reaparecen las visiones, pero en vez de aislarle y torturarle, le llevan de vuelta al grupo, reforzando la necesidad de cooperación con Furiosa y avisándole de los peligros que le esperan y cómo enfrentarse a ellos. Butler se pregunta:

¿Qué beneficio puede obtenerse del duelo, de prolongar la pena, de quedar expuestos a su carácter insoportable y no tratar de resolverlo por la vía de la violencia? [...] Si nos quedamos con el sentido de la pérdida, ¿vamos a sentirnos débiles y pasivos, como algo que debamos temer? ¿O por el contrario vamos a recuperar el sentido de la vulnerabilidad humana y a asumir una responsabilidad colectiva por las vidas físicas de los otros? (2006: 56)

Son precisamente estas cuestiones las que plantea Fury Road: el héroe de la saga reconoce su vulnerabilidad y acepta el duelo como una oportunidad de conectar con su entorno cuando descubre que éste no resulta necesariamente hostil, ya que es igual de vulnerable que él mismo. 
Butler señala que cuando se sufre una pérdida, ya sea de una persona concreta o de una comunidad, se revelan los lazos que unen entre sí a los individuos (2006: 48). La muerte de Angharad, la esposa embarazada, supone un punto de inflexión importante para la trama de Fury Road (y sucede en el punto medio exacto del filme). No se trata, pese a ello, de un momento determinante para el desarrollo de la acción (ya que no detiene la huida de las mujeres), sino que supone una ocasión de inclinación emocional para el grupo. Tras la pérdida de Angharad, tanto las mujeres como Furiosa y Max aceptan colaborar entre sí para perseguir sus objetivos. En la siguiente secuencia de enfrentamiento directo con el ejército de Joe, el vínculo formado entre Max y Furiosa se simboliza a través de sus pérdidas físicas: cuando Max pierde el equilibrio mientras lucha sobre el vehículo y cae de éste, Furiosa le salva cogiéndole de la pierna protésica con su brazo postizo. A través de aquello que sus enfrentamientos con la violencia en The Wasteland les han arrebatado, los personajes consiguen salvarse el uno al otro. Si bien en esta escena es Furiosa quien, pese a ser apuñalada consigue salvar a Max, la secuencia desemboca en el momento en que los roles se invierten y es él quien salva a Furiosa. Max utiliza la propia herramienta con la que Immortan Joe explotaba su cuerpo, la vía de trasfusión de sangre, para inyectar sangre a Furiosa y evitar su muerte. El protagonista se inclina literal y emocionalmente hacia Furiosa, presentada como cuerpo inerme que necesita del cuidado del héroe. Se trata de una unión física, que tiene como origen el reconocimiento por parte de Max de la vulnerabilidad de su propio cuerpo, ya no explotado violentamente por otros, pero sí al servicio de algo más allá de él mismo como individuo. En palabras de Butler, los otros no sólo quedan incorporados al límite que contiene al sujeto, sino que también le desintegran:

El duelo nos enseña la sujeción a la que nos somete nuestra relación con los otros en formas que no siempre podemos contar o explicar -formas que a menudo interrumpen el propio relato autoconsciente que tratamos de brindar, formas que desafían la versión de uno mismo como sujeto autónomo capaz de controlarlo todo. (2006: 49)

Así es la historia de Max, un relato del yo elaborado a partir del proceso del duelo y el descubrimiento de la sujeción que supone la relación con otros, que desintegran progresivamente la versión aislada e impermeable del protagonista que presentan las anteriores películas para entregarle, como ser corporal, a otros más allá de él mismo. "La autoridad narrativa del 'yo' debe ceder paso a la perspectiva y la temporalidad de un conjunto de normas que impugnan la singularidad de mi historia", afirma Butler (2009: 56). La historia de Max es la del duelo por las personas a las que ha perdido y aquellas otras que encuentra en su camino, que le conforman y deforman al mismo tiempo. Mientras transfiere sangre a Furiosa, vertiéndola literalmente de su cuerpo, revela algo que había ocultado hasta ese momento: su nombre. Al principio del filme, Furiosa se lo pregunta y él evita dar respuesta. Butler hace referencia a Adriana Cavarero cuando ésta afirma que para poder hablar de un "yo" debemos hacerlo en 
relación a un "tú": "sin el 'tú', mi propia historia resulta imposible" (Butler 2009: 50). Como propone Cavarero, el "yo" necesita estar fundamentalmente expuesto al otro para constituir la propia singularidad (2009: 51). Solo en el momento de máxima exposición de Max éste decide nombrarse a sí mismo ante Furiosa: “La singularidad del otro queda expuesta ante mí, pero la mía también se expone ante él" (2009: 52). De igual forma, Meri Torras y Michelle Gama hacen referencia al pensamiento de Butler cuando afirman que "pedir conocimiento u ofrecerlo no significa pedir que se reconozca lo que uno ya es. Significa invocar un devenir, instigar una transformación, exigir un futuro siempre en relación con el Otro" (2014: 72). Cuando Furiosa pregunta a Max por su nombre está pidiendo de él este devenir, aceptándole como parte de sí misma y del grupo, pero no recibe el reconocimiento de Max hasta el momento en que éste completa su transformación y expone su vulnerabilidad, revelándose a sí mismo a través de su nombre: "Max. My name is Max".

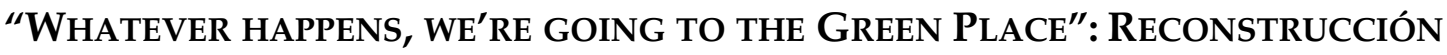 DE LA COMUNIDAD PERDIDA}

En Fury Road la experiencia común de la violencia y del duelo crea los vínculos entre los personajes. El duelo, como especifica Butler, no sólo hace referencia a la pérdida de una persona, sino que también se da por la pérdida de una comunidad. En el filme, el caso más obvio es el de Furiosa, personaje llevado por la rabia y el anhelo de venganza contra aquellos que la despojaron de su comunidad original, las Vuvalini. Pero Furiosa no es la única, ya que Max evoluciona durante la saga a la vez que vive la pérdida progresiva del mundo, la civilización que ya no existe. Los personajes encuentran en el duelo un lugar en común en el que construirse como grupo, siendo el reconocimiento de la vulnerabilidad, como afirman Torras y Gama, necesario "para volverse parte de un encuentro ético" (2014: 70). Pero el objetivo del grupo es alcanzar The Green Place of Many Mothers, la comunidad utópica en la que nació Furiosa cuando la tierra aún estaba viva. El horizonte de The Green Place esconde la utopía dentro de la distopía que es Fury Road, algo significativo en las distopías críticas de acuerdo con Rafaella Baccolini (2000) e Ildney Cavalcanti (2003). Ésta última afirma que "utopia in the feminist critical dystopia resides in the realm of the ineffable, invisible and silent" (Cavalcanti 2003: 50) y señala la contradicción inherente en el término, ya que la utopía se refiere a un lugar ideal que es, al mismo tiempo, un no lugar. The Green Place funciona precisamente como tal, siendo el objetivo al que aspirar que en realidad ya no existe.

Volviendo a las reflexiones de Butler en torno a la vulnerabilidad, es significativo cómo esta esperanza del grupo por recuperar una comunidad perdida, basada en unos valores no hipermasculinos, puede entenderse como la alternativa que la autora presenta a la violencia cuando plantea que 
tal vez exista otra forma de vida en la que uno no quede convertido emocionalmente en un muerto ni miméticamente en un violento, un modo de salir completamente del círculo de la violencia. Esta posibilidad se relaciona con la exigencia de un mundo donde la vulnerabilidad corporal esté protegida sin ser erradicada, subrayando la línea que separa a una de la otra. (Butler 2006: 70)

Efectivamente, Fury Road propone un devenir de su protagonista, Max, hacia la percepción, la aceptación y el reconocimiento de su vulnerabilidad a partir del duelo, lo que le permite establecer lazos con el resto de los personajes, principalmente Furiosa, y unirse a su lucha. El duelo ocupa un lugar crucial en la trama, como momento de revelación de la vulnerabilidad compartida. Como distopía crítica, la película dibuja The Green Place como lugar utópico inexistente, pero al mismo tiempo ofrece a los personajes el espacio en el que recobrar la esperanza por alcanzar algo cercano a ello, sustituyendo el mundo basado en valores hipermasculinos por una alternativa fundamentada en la vulnerabilidad como intrínseca a la condición humana. Furiosa busca al principio del filme el regreso al "no lugar" utópico que reside en su memoria, pero el regreso a éste la acaba llevando a la esperanza de reconstrucción de su lugar de partida, Citadel.

\section{BIBLIOGRAFÍA}

ARENDT, H. (2006), Sobre la violencia, Madrid, Alianza.

BACCOLINI, R. (2000), "Gender and Genre in the Feminist Critical Dystopias of Katharine Burdekin, Margaret Atwood, and Octavia Butler", en Future Females, The Next Generation: New Voices and Velocities in Feminist Science Fiction Criticism, Barr, M.S. (ed.), Lanham, Rowman \& Littlefield, 13-34.

BIBER, K. (2001), “The Threshold Moment: Masculinity at Home and on the Road in Australian Cinema", Limina, 7, 26-46.

BIRULÉS, F. (2007), "Reflexiones sobre vulnerabilidad y violencia”, en Violencia deliberada: Las raíces de la violencia patriarcal, Molas, M.D. (ed.), Barcelona, Icaria, 17-25.

BRAITHWAITE, A. (2011), “'It's the Beast Thing': Victimization, Violence and Popular Masculine Crisis", Feminist Media Studies, 11(4), 417-432.

BURK, L.R., BURKHART, B.R. y SIKORSKI, J.F. (2004), “Construction and Preliminary Validation of the Auburn Differential Masculinity Inventory", Psychology of Men $\mathcal{E}$ Masculinity, 5(1), 4-17.

BUTLER, J. (2002), Cuerpos que importan: Sobre los limites materiales y discursivos del sexo, Barcelona, Paidós.

BUTLER, J. (2006), Vida precaria: El poder del duelo y la violencia, Buenos Aires, Paidós.

BUTLER, J. (2007), El género en disputa: El feminismo y la subversión de la identidad, Barcelona, Paidós.

BUtLeR, J. (2009), Dar cuenta de sí mismo: Violencia ética y responsabilidad, Buenos Aires, Amorrortu.

CAVAlCANTI, I. (2003), “The Writing of Utopia and the Feminist Critical Dystopia: Suzy McKee Charnas's Holdfast Series", en Dark Horizons: Science Fiction and the 
Dystopian Imagination, Baccolini, R. y Moylan, T. (eds.), Nueva York, Routledge, 4768.

CR (2015), “Mad Max: Fury Road. Movie Production Notes" [en línea]. Cinema review, 2015. [Consulta: 17 febrero 2016]. Disponible en: $<$ http://www.cinemareview.com/production.asp?prodid=20022 $>$.

ConNelL, R. W. (2000), The Men and the Boys, Oakland, University of California Press.

Cornell, D. (2009), Clint Eastwood and Issues of American Masculinity, Nueva York, Fordham University Press.

FREITAS, C. (2015), “Finding Hope Without Salvation in Mad Max: Fury Road" [en línea]. Antipode Foundation, 21 agosto 2015 [Consulta: 9 marzo 2016]. Disponible en: $<$ https://antipodefoundation.org/2015/08/21/finding-hope-without-salvation/>.

HANLEY, G. (2010), Australian Legends: Historical Explorations of Australian Masculinity and Film 1970-1995, MA Thesis, University of Waikato [Consulta: 3 febrero 2016]. Disponible en: <http://researchcommons.waikato.ac.nz/handle/10289/4295>.

HEUSSER, J. (2015), “FXPodcast \#293: Mad Max: Fury Road” [en línea]. FXGuide, 29 mayo 2015 [Consulta: 17 febrero 2016]. Disponible en: $<$ https://www.fxguide.com/fxpodcasts/fxpodcast-293-mad-max-fury-road/>.

JefFORDS, S. (1994), Hard Bodies: Hollywood Masculinity in the Reagan Era, Nueva Jersey, Rutgers University Press.

MiLleR, G. (dir.) (1979), Mad Max, Australia, Roadshow Entertainment, American International Pictures, Warner Bros.

MilleR, G. (dir.) (1981), Mad Max 2, Australia, Warner Bros.

Miller, G. (dir.) (1985), Mad Max Beyond Thunderdome, Australia, Warner Bros.

MiLleR, G. (dir.) (2015), Mad Max: Fury Road, Australia/EEUU, Village Roadshow Pictures.

SAEZ TAJAFUERCE, B. (ed.) (2014), Cuerpo, memoria y representación: Adriana Cavarero y Judith Butler en diálogo, Barcelona, Icaria.

SCHARRER, E. (2001), “Tough Guys: The Portrayal of Hypermasculinity and Aggression in Televised Police Dramas", Journal of Broadcasting \& Electronic Media, 45(4), 61534.

SHARRETT, C. (1985), “The Hero as Pastiche: Myth, Male Fantasy, and Simulacra in Mad Max and The Road Warrior", Journal of Popular Film and Television, 2(13), 80-91.

SIEGEL, D. (dir.) (1971), Dirty Harry, EEUU, The Malpaso Company.

TASKER, Y. (1995), Spectacular Bodies: Gender, Genre and the Action Cinema, PhD Thesis, University of Warwick [Consulta: 22 enero 2016]. Disponible en: $<$ http://webcat.warwick.ac.uk/record=b1399932 S1>.

TORRAS FRANCÉS, M., y GAMA LEYVA, M. (2014), “Un diálogo entre Judith Butler y Adriana Cavarero (Itinerario de resonancias)", en Cuerpo, memoria y representación: Adriana Cavarero y Judith Butler en diálogo, Begonya Saez Tajafuerce (ed.), Barcelona, Icaria, 99-120.

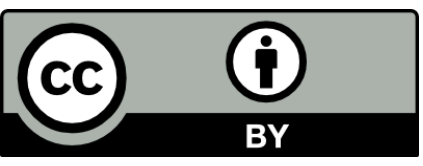

Llevat que s'hi indiqui el contrari, els continguts d'aquesta revista estan subjectes a la llicència de Creative Commons: Reconeixement 3.0 Espanya. 